\title{
THE RENORMALIZED VOLUME AND THE VOLUME OF THE CONVEX CORE OF QUASIFUCHSIAN MANIFOLDS
}

\author{
JEAN-MARC SCHLENKER
}

\begin{abstract}
We show that the renormalized volume of a quasifuchsian hyperbolic 3-manifold is equal, up to an additive constant, to the volume of its convex core. We also provide a precise upper bound on the renormalized volume in terms of the WeilPetersson distance between the conformal structures at infinity. As a consequence we show that holomorphic disks in Teichmüller space, which are large enough must have "enough" negative curvature.
\end{abstract}

\section{Results}

1.1. Notations. In all the paper, we consider a closed surface $S$ of genus $g \geq 2$, and we call $\mathcal{T}_{S}$ the Teichmüller space of $S$. Given a complex structure $c \in \mathcal{T}_{S}$, we denote by $Q_{c}$ the vector space of holomorphic quadratic differentials on $(S, c)$. We also call $\mathcal{G}_{S}$ the space of quasifuchsian metrics on $S \times \mathbb{R}$, considered up to isotopy.

The Bers Simultaneous Uniformization Theorem provides a homeomorphism between $\mathcal{T}_{S \cup \bar{S}}$ and $\mathcal{G}_{S}$. So $\mathcal{G}_{S}$ is parameterized by $\mathcal{T}_{+} \times \mathcal{T}_{-}$, where $\mathcal{T}_{+}$and $\mathcal{T}_{-}$are two copies of $\mathcal{T}_{S}$ corresponding respectively to the upper and lower boundaries at infinity of $S \times \mathbb{R}$.

For $q \in \mathcal{G}$, we denote by $V_{R}(q)$ the renormalized volume of $(S \times \mathbb{R}, g)$ (as defined in Section 3 following, e.g., [16]), while $C(q)$ is the convex core of $(S \times \mathbb{R}, g)$ and $V_{C}(q)$ is its volume.

1.2. Comparing the renormalized volume to the volume of the convex core. The first result presented here is a precise comparison between the volume of the convex core and the renormalized volume of a quasifuchsian hyperbolic manifold.

Theorem 1.1. There exists a constant $C_{g}>0$, depending only on the genus $g$ of $S$, as follows. Let $q \in \mathcal{G}_{S}$ be a quasifuchsian metric. Then

$$
V_{R}(q) \leq V_{C}(q)-(1 / 4) L_{m}(l) \leq V_{R}(q)+C_{g},
$$

where $l$ is the measured bending lamination of the boundary of $C(q), m$ is its induced metric, and $L_{m}(l)$ is the length of $l$ with respect to $m$. Equality in the first inequality occurs exactly when $q$ is Fuchsian.

Note that the the quantity $V_{C}(q)-(1 / 4) L_{m}(l)$ appearing in this theorem can be interpreted as the half-sum of the volume and the "dual volume" of the convex core (as appearing e.g. in [15]). Recall also that there is a bound on $L_{m}(l)$ depending only on the genus $g$; see [2].

Received by the editors March 5, 2012. 
Theorem 1.1 can be extended to convex co-compact manifolds with incompressible boundary. We do not elaborate on this here but the statement and proof of the extension should appear clearly from the proof of Theorem 1.1 below.

1.3. An upper bound on the renormalized volume. The next result is a precise upper bound on the renormalized volume of a quasifuchsian manifold, in terms of the Weil-Petersson distance between its conformal structures at infinity.

Theorem 1.2. For any quasifuchsian metric $q$ on $S \times \mathbb{R}$,

$$
V_{R}(q) \leq 3 \sqrt{\pi(g-1)} d_{W P}\left(c_{-}, c_{+}\right),
$$

where $c_{-}$and $c_{+}$are the conformal structure at infinity of $q$ and $d_{W P}$ is the WeilPetersson distance.

This result is related, through Theorem 1.1, to the following result of Brock on the comparison between the Weil-Petersson distance $d_{W P}\left(c_{-}, c_{+}\right)$and the volume of the convex core $V_{C}(q)$.

Theorem 1.3 (Brock [3]). For a given surface $S, V_{C}\left(c_{-}, c_{+}\right)$is comparable to $d_{W P}\left(c_{-}, c_{+}\right)$, that is, there are constants $k_{1}, k_{2}>0$ such that

$$
\frac{d_{W P}\left(c_{-}, c_{+}\right)}{k_{1}}-k_{2} \leq V_{C}\left(c_{-}, c_{+}\right) \leq k_{1} d_{W P}\left(c_{-}, c_{+}\right)+k_{2} .
$$

We will recover a precise form of the upper bound on $V_{C}$ from Theorems 1.1 and 1.2. Recall that Bridgeman [2, Proposition 2] proved that the length of the measured bending lamination, $L_{m}(l)$, is bounded from above by a constant $K_{g}$ depending only on the genus of $S$. The following statement then follows immediately from Theorems 1.2 and 1.1 .

Corollary 1.4. There exists a constant $K_{g}$ depending only on the genus of $S$ such that

$$
V_{C}\left(c_{-}, c_{+}\right) \leq 3 \sqrt{\pi(g-1)} d_{W P}\left(c_{-}, c_{+}\right)+K_{g}
$$

This is a more precise version of the second inequality in (1.2).

1.4. Holomorphic disks in Teichmüller space. An interesting difference between Theorems 1.2 and 1.3 is that the renormalized volume has, in addition to the "coarse" properties of the volume of the convex core, some remarkable analytic properties related to the Weil-Petersson metric on Teichmüller space. We will use this and the upper bound on $V_{C}$ to obtain a statement on the global geometry of the WeilPetersson metric on Teichmüller space, more precisely on the maximal radius of holomorphic disks with curvature bounded from below (see Theorem 1.5). The statement below is probably not optimal, it is more of an indication of the type of results one can obtain using the remarkable properties of the renormalized volume.

Theorem 1.5. There exists a smooth, increasing function $\phi:[0,1) \rightarrow \mathbb{R}_{\geq 0}$ with $\phi(0)=0, \phi^{\prime}(0)=2$ and $\lim _{1} \phi=\infty$ as follows. Let $k>0$ be such that $3 k^{2} \sqrt{\pi(g-1)}<$ 2. There is no immersed holomorphic disk $D$ of radius $\phi\left(3 k^{2} \sqrt{\pi(g-1)} / 2\right) / k$ in $\mathcal{T}_{S}$ with curvature $K \geq-k^{2}$. 
The radius here is the radius of the metric induced on $D$ by the Weil-Petersson metric on $\mathcal{T}_{S}$. The expression of the function $\phi$ can be obtained by solving a simple differential equation; see Section 6 . This kind of statement is presumably most interesting close to the boundary of $\mathcal{T}_{S}$ for the Weil-Petersson metric, where the sectional curvature tends to be close to 0 in many directions; see [25, 24]. It should be compared to (and is related to) the isoperimetric inequality for complex submanifolds of Teichmüller space discovered by McMullen; see [17, Corollary 1.3].

1.5. From the boundary of the convex core to infinity. There is a clear parallel between data "at infinity" of a quasifuchsian (or more generally a convex co-compact hyperbolic manifold) and corresponding data on the boundary of the convex core. The results presented here contribute to clarify and extend those analogies. A synthetic presentation of this correspondence is shown in Table 1. Clearly, the conformal metric at infinity corresponds to the induced metric on the boundary of the convex core. The analog at infinity of the measured bending lamination of the boundary of the convex core is less obvious, but it appears to be the "second fundamental form at infinity" $I^{*}$ as defined in Section 3.4, or more precisely its traceless part $\Pi_{0}^{*}$. This second fundamental form at infinity gives the second term in the asymptotic expansion of the metric at infinity associated to an equidistant foliation, see below, and in this sense, too, it is analogous to the measured bending lamination on the boundary of the convex core. Other elements in this analogy between the data on the boundary of the convex core and the data at infinity are resumed in Table 1, the different lines are explained below.

\section{Conformal changes of metrics}

We gather in this short section some basic and well-known results on conformal changes of metrics on surfaces.

Lemma 2.1. Let $h$ be a Riemannian metric on $S$, of curvature $K$. Let $u: S \rightarrow \mathbb{R}$, let $\bar{h}=e^{2 u} h$, and let $\bar{K}$ be the curvature of $\bar{h}$. Then

$$
\bar{K}=e^{-2 u}(K+\Delta u) .
$$

Here $\Delta$ is the "geometer's" Laplacian, that is, it is non-positive at the minima. The proof of this lemma can be found, e.g., in [1, Section 1]

Lemma 2.2. Let $h$ be a hyperbolic metric on $S$, and let $u: S \rightarrow \mathbb{R}$ be such that $e^{2 u} h$ has curvature $\bar{K} \geq-1$. Then $u \geq 0$.

TABLE 1. Infinity versus the boundary of the convex core.

\begin{tabular}{ll}
\hline Boundary of the convex core & At infinity \\
\hline Induced metric $m$ & Conformal/hyperbolic metric at infinity \\
Measured bending lamination $l$ & $\Pi_{0}^{*}$ \\
Bound on $L_{m}(l)[2]$ & Theorem 5.1 \\
Volume of the convex core & Renormalized volume \\
Bonahon's Schläfli formula & Proposition 3.10 \\
Brock's upper bound on $V_{C}[3]$ & Theorem 1.2 \\
\hline
\end{tabular}


Proof. Let $x \in S$ be a point where $u$ is minimal. Then $\Delta u \leq 0$ at $x$, and $\bar{K}=$ $e^{-2 u}(-1+\Delta u) \leq-e^{-2 u}$. Since $\bar{K} \geq-1$ it follows that $u \geq 0$ at $x$.

\section{The renormalized volume}

3.1. Some background. Consider a Poincaré-Einstein manifold $M$, that is, a manifold $M$ with boundary, with an Einstein metric $g$ on the interior of $M$ which can be written as

$$
g=\frac{\bar{g}}{\rho^{2}}
$$

where $\rho$ is a function which vanishes on $\partial M$ with $\|d \rho\|_{\bar{g}}=1$ on $\partial M$.

The volume of $(M, g)$ is infinite, however there is a well-defined way to define a "regularized" version of this volume, called the renormalized volume of $M$, which is finite (see, e.g., $[8,10]$ ). If the dimension of $M$ is odd, it depends on the choice of a metric in the conformal class of the boundary of $(M, g)$, while if the dimension of $M$ is even it is canonically defined.

If $M$ is a convex co-compact hyperbolic manifold, it is Poincaré-Einstein according to the definition above, so that the definition of its renormalized volume applies. The fact that the metric has constant curvature makes it possible to give an explicit description of the geometry of the leaves of an equidistant foliation, see [18]. Since the dimension is odd, this renormalized volume depends on the choice of a metric at infinity, however there is a canonical choice available for this metric: the unique metric of constant curvature -1 in the conformal class at infinity.

This renormalized volumes turns out to be strongly related to the Liouville functional previously studied by Takhtajan and Zograf [21], and Takhtajan and Teo [22], see [13]. In particular it has some remarkable relations to the Weil-Petersson metric on Teichmüller space. Moreover, there is a simpler definition specific to dimension 3. We recall below this definition and the key properties of this three-dimensional renormalized volume in a form suitable for the applications considered here.

We can mention that the renormalized volume is the real part of a complex quantity with an imaginary part related to the Chern-Simons invariant, see [11]. Some of the properties of the three-dimensional renormalized volume used here actually extend in some measure to higher (odd) dimensions; see [12].

3.2. Metrics at infinity and equidistant foliations. We now consider a quasifuchsian hyperbolic 3-manifold $(M, g)$.

Definition 3.1. Let $E$ be an end of $M$. An equidistant foliation in $E$ is a foliation of a neighborhood of infinity in $E$ by convex surfaces, $\left(S_{r}\right)_{r \geq r_{0}}$, for some $r_{0}>0$, such that, for all $r^{\prime}>r \geq r_{0}, S_{r^{\prime}}$ is between $S_{r}$ and infinity, and at constant distance $r^{\prime}-r$ from $S_{r}$.

Two equidistant foliations in $E$ will be identified if they coincide in a neighborhood of infinity. In this case, they can differ only by the first value $r_{0}$ at which they are defined.

Note that given an equidistant foliation $\left(S_{r}\right)_{r} \geq r_{0}$ and given $r^{\prime}>r \geq 0$, there is a natural identification between $S_{r}$ and $S_{r^{\prime}}$, obtained by following the normal direction from $S_{r}$ to $S_{r^{\prime}}$. This identification will be implicitly used below. 
Definition 3.2. Let $M$ be a convex co-compact hyperbolic manifold, let $E$ be an end of $M$, and let $\left(S_{r}\right)_{r \geq r_{0}}$ be an equidistant foliation in E. The metric at infinity associated to $\left(S_{r}\right)_{r \geq r_{0}}$ is the metric:

$$
I^{*}=\lim _{r \rightarrow \infty} \mathrm{e}^{-2 r} I_{r}
$$

where $I_{r}$ is the induced metric on $S_{r}$.

We will make use of the following proposition. The first part is quite elementary (see e.g. [14]) while the second part can be found, in the more general setting of conformally compact Einstein manifolds, in [8, Lemma 2.1] (see also [9, Lemma 5.2] and [4, Lemma 2.1.2]).

Proposition 3.3. $I^{*}$ always exists, and it is in the conformal class at infinity of E.

Let $M$ be a convex co-compact hyperbolic manifold, let $E$ be an end of $M$, and let $h$ be a Riemannian metric in the conformal class at infinity of E. There is a unique equidistant foliation in $E$ such that the associated metric at infinity is $h$.

This equidistant foliation can be defined from $I^{*}$ in terms of envelope of a family of horospheres, see [6], we recall this construction here. Consider the hyperbolic space $H^{3}$ as the universal cover of $M$, then $I^{*}$ lifts to a metric on the domain of discontinuity $\Omega$ of $M$, in the canonical conformal class of $\partial_{\infty} H^{3}$. Let $x \in \Omega$. For each $y \in H^{3}$, the visual metric $h_{y}$ on $\partial_{\infty} H^{3}$ is conformal to $I^{*}$. Let $H_{x, r}$ be the set of points $y \in H^{3}$ such that $h_{y} \geq e^{2 r} I^{*}$ at $x$ - it is not difficult to check that $H_{x, r}$ is a horoball intersecting $\partial_{\infty} H^{3}$ at $x$, and the lift of $S_{r}$ to $H^{3}$ happens to be equal to the boundary of the union of the $H_{x, r}$, for $x \in \Omega$.

3.3. Definition and first variation of $\boldsymbol{W}$. To define the renormalized volume of a quasifuchsian manifold, below, we first introduce a modified volume of convex subsets. We consider a quasifuchsian manifold $M$ and a convex subset $N$ of $M$ with smooth boundary - here "convex" means that, whenever $\gamma \subset M$ is a geodesic segment with endpoints in $N, \gamma \subset N$. We will define first (in Definition 3.4) a modified volume of $N$, and then use this modified volume, for a particular choice of a convex subset of $M$, to define the renormalized volume of $M$ (Definition 3.9).

Definition 3.4. Let $N \subset M$ be a convex subset. We define

$$
W(N)=V(N)-\frac{1}{4} \int_{\partial N} H d a
$$

where $H$ is the mean curvature of $\partial N$ and $d a$ is the area form of its induced metric.

The first variation of this modified volume is given in [14], based on an earlier variation formula for deformations of Einstein manifolds with boundary $[20,19]$. Here we consider a first-order deformation of the hyperbolic metric on $N$, and denote by $I^{\prime}$ and $I^{\prime}$, respectively, the corresponding first-order variations of the induced metric and second fundamental form on the boundary of $N$.

Lemma 3.5. Under a first-order deformation of $N$,

$$
W^{\prime}=\frac{1}{4} \int_{\partial N}\left\langle I^{\prime}-\frac{H}{2} I^{\prime}, I\right\rangle d a .
$$


The scalar product appearing in (3.1) between symmetric bilinear forms is the usual extension to tensors of the Riemannian scalar product on $T \partial N$ associated to the induced metric $I$.

The following lemma is a direct consequence of Lemma 3.5, see below.

Lemma 3.6. Let $r \geq 0$, let $N_{r}$ be the set of points of $M$ at distance at most $r$ from $N$. Then $W\left(N_{r}\right)=W(N)-\pi r \chi(\partial M)$.

Proof. For $s \in[0, r]$, let $N_{s}$ be the set of points of $M$ at distance at most $s$ from $N$, and let $w(s)=W\left(N_{s}\right)$. Let $I_{s}, I_{s}, I I I_{s}$ and $B_{s}$ be the induced metric, second and third fundamental forms and the Weingarten operator of $\partial N_{s}$. According to standard differential geometry formulas,

$$
I_{s}^{\prime}=2 I_{s}, \quad \Pi_{s}^{\prime}=I I_{s}+I_{s} .
$$

Lemma 3.5 therefore shows that

$$
\begin{aligned}
W\left(N_{s}\right)^{\prime} & =\frac{1}{4} \int_{\partial N}\left\langle I I I_{s}+I_{s}-H_{s} I_{s}, I_{s}\right\rangle d a_{s}=\frac{1}{4} \int_{\partial N} \operatorname{tr}\left(B_{s}^{2}\right)+2-H_{s}^{2} d a_{s} \\
& =\frac{1}{4} \int_{\partial N} 2-2 \operatorname{det}\left(B_{s}\right) d a_{s}=\frac{1}{2} \int_{\partial N}-K d a_{s}=-\pi \chi(\partial N) .
\end{aligned}
$$

Consider a Riemannian metric $h$ on $\partial M$ in the conformal class at infinity of $M$. By Proposition 3.3 there is a unique equidistant foliation $\left(s_{r}\right)_{r}>_{0}$ of $M$ near infinity such that the associated metric is $h$. For $r$ large enough, the surfaces $S_{r}$ bound a convex subset of $M$, so hat Definition 3.4 can be applied.

Definition 3.7. Let $h$ be a metric on $\partial M$, in the conformal class at infinity. Let $\left(S_{r}\right)_{r \geq r_{0}}$ be the equidistant foliation at infinity associated to $h$. We define $W(M, h):=$ $W\left(S_{r}\right)+\pi r \chi(\partial M)$, for any choice of $r \geq r_{0}$.

This definition does not depend on the choice of $r$ by Lemma 3.6.

Corollary 3.8. For any $\rho \in \mathbb{R}, W\left(M, e^{2 \rho} h\right)=W(M, h)-\pi \rho \chi(\partial M)$.

3.4. Variational formula for $W$ from infinity. Given an equidistant foliation of the end $E$, the hyperbolic metric $q$ actually takes a remarkably simple form; see $[14,16]$. It can be written as

$$
q=d r^{2}+e^{2 r} I^{*}+2 I^{*}+e^{-2 r} \Pi^{*},
$$

where $I^{*}$ is the metric at infinity called $h$ above, and $I^{*}$ and $I^{*}$ are analogs at infinity of the second and third fundamental forms of a surface. More precisely, there is a unique bundle morphism $B^{*}: T S \rightarrow T S$ which is self-adjoint for $I^{*}$ and such that

$$
I^{*}=I^{*}\left(B^{*} \cdot, \cdot\right), \quad I^{*}=I^{*}\left(B^{*} \cdot, B^{*} \cdot\right) .
$$

Then $B^{*}$ satisfies the Codazzi equation $d^{\nabla^{*}} B^{*}=0$, where $\nabla^{*}$ is the Levi-Civita connection of $I^{*}$, and an analog of the Gauss equation, $\operatorname{tr}\left(B^{*}\right)=-K^{*}$, where $K^{*}$ is the curvature of $I^{*}$.

Consider now, as in Section 3.3, a convex subset $N \subset M$ with smooth boundary, and the equidistant foliation of $M \backslash N$ by surfaces at constant distance from $N$. The data at infinity $I^{*}, I^{*}, I^{*}$ can be written in terms of the data $I, I I, I I$ on the boundary 
of $N$ as follows (see [14, Section 5]): if $E$ is the identity on $T \partial N$ and $B$ is the shape operator of $T N$, then

$$
\begin{aligned}
I^{*} & =\frac{1}{2} I((E+B) \cdot,(E+B) \cdot), \quad I^{*}=\frac{1}{2} I((E+B) \cdot,(E-B) \cdot), \\
\text { III }^{*} & =\frac{1}{2} I((E-B) \cdot,(E-B) \cdot) .
\end{aligned}
$$

Conversely, a direct computation (see also [14, Section 5]) shows that the same formulas express the data on the boundary of $N$ in terms of the data at infinity:

$$
\begin{aligned}
I & =\frac{1}{2} I^{*}\left(\left(E+B^{*}\right) \cdot,\left(E+B^{*}\right) \cdot\right), \quad I I=\frac{1}{2} I^{*}\left(\left(E+B^{*}\right) \cdot,\left(E-B^{*}\right) \cdot\right), \\
\text { III } & =\frac{1}{2} I^{*}\left(\left(E-B^{*}\right) \cdot,\left(E-B^{*}\right) \cdot\right) .
\end{aligned}
$$

Using those transformation formulas, one can write the first-order variation of $W$ in terms of the data at infinity, and it turns out to be remarkably similar to the variation formula (3.1) in terms of the data on $\partial N$, see [14, Section 5].

$$
W^{\prime}=-\frac{1}{4} \int_{\partial N}\left\langle I^{* \prime}-\frac{H^{*}}{2} I^{* \prime}, I^{*}\right\rangle d a^{*} .
$$

Here $I^{* \prime}$ and $I^{* \prime}$ are the first-order variations of $I^{*}$ and $I^{*}$, while $H^{*}=\operatorname{tr}\left(B^{*}\right)$ and $d a^{*}$ is the area form of $I^{*}$.

3.5. The renormalized volume. We can now give the definition of the renormalized volume of $M$.

Definition 3.9. The renormalized volume $V_{R}$ of $M$ is defined as equal to $W(h)$ when the metric at infinity $h$ is the unique metric of constant curvature -1 in the conformal class at infinity of $M$.

Another possible definition is as the maximum of $W(M, h)$ over all metrics $h$ in the conformal class at infinity of $M$, under the condition that the area of $h$ is equal to $-2 \pi \chi(\partial M)$; see [14].

3.6. A variational formula for the renormalized volume. Consider now a first-order deformation of $M$, specified - through the Bers Double Uniformization Theorem - by a first-order deformation of the conformal structure at infinity, considered as a point in the Teichmüller space of $\partial M$.

Proposition 3.10. Under a first-order deformation of the hyperbolic structure on $M$,

$$
d V_{R}=-\frac{1}{4}\left\langle I_{0}^{*}, \dot{I}^{*}\right\rangle
$$

Here $\langle$,$\rangle is the duality bracket, \Pi_{0}^{*}$ is considered as the real part of a holomorphic quadratic differential, and therefore a vector in the cotangent bundle of $\mathcal{T}$. 


\subsection{Comparing metrics at infinity.}

Proposition 3.11. If $h, h^{\prime}$ are two metrics of non-positive curvature in the conformal class at infinity on $\partial M$ and $h^{\prime}$ is everywhere at least as large as $h$, then $W\left(M, h^{\prime}\right) \geq$ $W(M, h)$, with equality if and only if $h=h^{\prime}$.

The proof of this proposition will follow the next two lemmas.

Lemma 3.12. Let $h, h^{\prime}$ be two metrics in the conformal class at infinity on $\partial M$. Suppose that $h^{\prime}$ is everywhere at least as large as $h$. Let $r$ be large enough so that both $S_{h, r}$ and $S_{h^{\prime}, r}$ are well-defined. Then $S_{h, r}$ is in the interior of $S_{h^{\prime}, r}$.

Proof. We have seen above (just after Proposition 3.3) that $S_{h, r}$ can be defined as the boundary of the complement of the union of the horoballs associated to $h$ of "radius" $r$ at points of $\partial M$. Since $h^{\prime}$ is everywhere at least as large as $h$, the horoball associated to $h^{\prime}$ of radius $r$ is at each point contained in the horoball associated to $h$ of radius $r$. It follows that $M_{h, r} \subset M_{h^{\prime}, r}$.

Definition 3.13. Let $E$ be a hyperbolic end, let $S, S^{\prime}$ be two surfaces in $E$ such that $S$ is contained in the "interior" of $S^{\prime}$. We set

$$
W\left(S, S^{\prime}\right)=V\left(S, S^{\prime}\right)-\frac{1}{4} \int_{S^{\prime}} H d a+\frac{1}{4} \int_{S} H d a .
$$

It follows from this definition that if $E$ is an end of $M$ containing two surfaces $S$ and $S^{\prime}$ with $S$ contained in the interior of $S^{\prime}$, if $h$ is the metric at infinity in the conformal class at infinity on $\partial M$ corresponding to $S$ in $\partial E$ and $h^{\prime}$ is another metric in the conformal class at infinity on $\partial M$, equal to $h$ except that it corresponds to $S^{\prime}$ in $\partial E$, then

$$
W\left(M, h^{\prime}\right)=W(M, h)+W\left(S, S^{\prime}\right) .
$$

Lemma 3.14. If $S$ is contained in the interior of $S^{\prime}$ and the induced metrics on both $S$ and $S^{\prime}$ have non-positive curvature, then $W\left(S, S^{\prime}\right) \geq 0$, with equality only if $S=S^{\prime}$.

Proof. We first construct a smooth one-parameter family of surfaces, $\left(S_{t}\right)_{t \in[0,1]}$, with $S_{0}=S, S_{1}=S^{\prime}$, and such that, for $t \leq t^{\prime}, S_{t}$ is contained in the interior of $S_{t^{\prime}}$ and that $S_{t}$ has an induced metric of non-positive curvature.

For this we will use the fact that given a surface $S \subset M$ is associated to a metric $h$ in the conformal class at infinity, then the curvature of $h$ has the same sign as the curvature of $S$ at the corresponding point (the correspondence being through the hyperbolic Gauss map). This is because the curvature of $h$ is equal to $K_{h}=\frac{K}{\operatorname{det}(E+B)}$, where $K$ is the curvature of the induced metric on $S$ (see [14][Lemma 5.2]) and $\operatorname{det}(E+B) \geq 0$ if $S$ corresponds to a metric $h$ at infinity.

Now consider the metrics at infinity $h, h^{\prime}$ corresponding to $S, S^{\prime}$ respectively. They are conformal and $h \leq h^{\prime}$ at each point by Lemma 3.12, so we can write $h^{\prime}=e^{2 u} h$ for a function $u: \partial M \rightarrow \mathbb{R}_{\geq 0}$. Then $K_{h^{\prime}}=e^{-2 u}\left(K_{h}+\Delta u\right)$ and both $K_{h}$ and $K_{h^{\prime}}$ are non-positive, so $K_{h} \leq 0$ and $K_{h}+\Delta u \leq 0$. For all $t \in[0,1]$ consider the metric $h_{t}=e^{2 t u} h$. It is conformal to $h$ and $h^{\prime}$, with curvature $K_{h_{t}}=e^{-2 t u}\left(K_{h}+t \Delta u\right) \leq 0$. So $h_{t}$ corresponds to a surface $S_{t}$ with non-positive curvature. The monotonicity of $\left(h_{t}\right)$ and Lemma 3.12 show that the $S_{t}$ provide a foliation of the domain of $M$ between $S$ and $S^{\prime}$, as required. 
It is now sufficient to prove that

$$
\frac{d}{d t} W\left(S, S_{t}\right) \geq 0
$$

with equality only if $S_{t}$ is stationary.

Consider now a fixed value of $t$, and suppose that the normal first-order deformation of $S_{t}$ is given by $u N$, where $N$ is the unit exterior normal to $S_{t}$ and $u$ is a non-negative function on $S_{t}$. We know (see [14, equation (41)]) that the first-order variation of $W\left(S_{t}\right)$ is given by

$$
\delta W\left(S_{t}\right)=\frac{1}{4} \int_{S_{t}} \delta H+\left\langle\delta I, \quad I I-\frac{H}{2} I\right\rangle d a,
$$

where $\delta H$ is the first-order variation of $H$ and $\delta I$ is the first-order variation of the induced metric.

Now a direct and classical computation shows that

$$
\delta I I=-H e s s(u)+u(I I I+I),
$$

while

$$
\delta I=2 u I I
$$

It follows that

$$
\delta H=\operatorname{tr}_{I}(\delta I I)-\langle\delta I, I\rangle=\Delta u+2 u-u \operatorname{tr}_{I}(I I I) .
$$

Therefore

$$
\delta W\left(S_{t}\right)=\frac{1}{4} \int_{S_{t}} \Delta u+2 u+\langle u I I, I I-H I\rangle d a,
$$

so

$$
\delta W\left(S_{t}\right)=\frac{1}{4} \int_{S_{t}} \Delta u+2 u-2 u \operatorname{det}(B) d a=\frac{1}{4} \int_{S_{t}} \Delta u-2 u K d a .
$$

But the integral of $\Delta u$ is zero while the other term is non-negative, and the result follows.

The proof of Proposition 3.11 clearly follows from this lemma.

Remark 3.15. Sergiu Moroianu pointed out that a simpler proof of Proposition 3.11 can be obtained once one knows that $W$ satisfies a "Polyakov formula" as in [12][(1), p.2], a fact that we do not use or prove here but which is true.

\section{Proof of Theorem 1.1}

4.1. The upper bound on $\boldsymbol{V}_{\boldsymbol{R}}$. Let $h_{g r}$ be the "grafting metric" on $\partial_{\infty} M$ (it is also sometimes called the Thurston metric). Recall that $h_{g r}$ is a metric with curvature in $[-1,0]$ in the conformal class at infinity. In the simplest case where the support of $l$ is a simple closed curve $c$, with a weight $w, h_{g r}$ is obtained by cutting $(\partial M, m)$ along the geodesic realizing $c$ and gluing in a flat strip of width $w$.

Lemma 4.1. Let $m$ and $l$ be the induced metric and the measured bending lamination on the boundary of the convex core of $M$. Then

$$
W\left(M, h_{g r}\right)=V_{C}(M)-\frac{1}{4} L_{m}(l) .
$$


Let $h_{0}$ be the hyperbolic metric in the conformal class at infinity of $M$. It follows from Lemma 2.2 that $h_{g r} \geq h_{0}$ at all points of $\partial_{\infty} M$, and Proposition 3.11 therefore indicates that $W\left(M, h_{0}\right) \leq W\left(M, h_{g r}\right)$. Since $V_{R}(M)=W\left(M, h_{0}\right)$, we find that $V_{R}(M) \leq V_{C}(M)-L_{m}(l) / 4$.

4.2. The lower bound on $\boldsymbol{V}_{\boldsymbol{R}}$. The area of $h_{g r}$ is equal to $-2 \pi \chi(\partial M)+L_{m}(l)$. Therefore, the metric

$$
h_{g r}^{\prime}:=\frac{-2 \pi \chi(\partial M)}{-2 \pi \chi(\partial M)+L_{m}(l)} h_{g r}
$$

has area equal to $-2 \pi \chi(\partial M)$, which is equal to the area of $h_{0}$. Since $V_{R}(M)$ is the maximum over $W(M, h)$ for $h$ a metric in the conformal class at infinity of area equal to the area of $h_{0}($ see $[14,16])$ we find that

$$
W\left(M, h_{g r}^{\prime}\right) \leq V_{R}(M) .
$$

However Corollary 3.8 indicates that

$$
W\left(M, h_{g r}^{\prime}\right)=W\left(M, h_{g r}\right)-\pi \log \left(\frac{-2 \pi \chi(\partial M)}{-2 \pi \chi(\partial M)+L_{m}(l)}\right) \chi(\partial M) .
$$

It is also known that, if $M$ has incompressible boundary, then $L_{m}(l) \leq C(M)$. It follows that

$$
W\left(M, h_{g r}\right) \leq V_{R}(M)+C^{\prime}(M),
$$

where $C^{\prime}(M)$ is a constant which can easily be explicitly computed in terms of $C(M)$ and of $\chi(\partial M)$. This concludes the proof of Theorem 1.1 .

\section{Proof of Theorem 1.2}

5.1. The Bers embedding. We recall here a the basic setup of the Bers embedding. We consider a quasifuchsian hyperbolic 3-manifold $M \simeq S \times \mathbb{R}$, denote by $c_{+}$and $c_{-}$ the complex structures at $+\infty$ and $-\infty$, respectively, and by $\sigma_{+}$and $\sigma_{-}$the complex projective structures at infinity. We also call $\sigma_{+}^{F}, \sigma_{-}^{F}$ the Fuchsian complex projective structures with underlying complex structures $c_{+}, c_{-}$on $S$. We can then define two holomorphic quadratic differentials

$$
q_{-}=\sigma_{-}-\sigma_{-}^{F}, \quad q_{+}=\sigma_{+}-\sigma_{+}^{F},
$$

where the minus sign refers to the comparison of two complex projective structures on a given Riemann surface using the Schwarzian derivative; see [5].

Then, if $q$ is the holomorphic quadratic differential on $(\partial M, c)$ corresponding to $q_{ \pm}$ on the corresponding boundary component of $M$, we have (see [14, Lemma 8.3])

$$
\Pi_{0}^{*}=-\operatorname{Re}(q),
$$

that is, the real part of $q$ is minus the traceless part of the second fundamental form at infinity.

We now fix the conformal structure $c_{-}$on the lower boundary at infinity of $M$, and vary $c_{+}$. Each choice of $c_{+}$determines a complex projective structure $\sigma_{-}$on the lower boundary at infinity of $M$, and therefore a holomorphic quadratic differential $q_{-} \in Q_{c_{-}}$. This defines a map $B_{+}: \mathcal{T}_{+} \rightarrow Q_{c_{-}}$, called the Bers embedding.

Using the hyperbolic metric $h_{-}$in the conformal class of $c_{-}$, we can measure at each point of $S$ the norm of $q_{-}$. We call $Q_{c_{-}}^{\infty}$ the vector space $Q_{c_{-}}$, endowed with this $L^{\infty}$ norm. 
Theorem 5.1 (Nehari). The image of $B_{+}$contains the ball of radius 2 , and is contained in the ball of radius 6 in $Q_{c_{-}}^{\infty}$.

See [7, Theorem 1, p. 134].

Consider now on $Q_{c_{-}}$the $L^{2}$-norm for the Weil-Petersson metric, and denote by $Q_{c_{-}}^{2}$ the vector space $Q_{c_{-}}$endowed with this norm.

Corollary 5.2. The image of $B_{+}$is contained in the ball of radius $12 \sqrt{\pi(g-1)}$ in $Q_{c_{-}}^{2}$.

5.2. The upper bound. We now prove Theorem 1.2. Let $c_{-}, c_{+} \in \mathcal{T}$, and let $d=d_{W P}\left(c_{-}, c_{+}\right)$.

With the notations introduced here, Proposition 3.10 can be written as follows. Recall that $Q_{c_{-}}$is naturally identified with the complex cotangent space of $\mathcal{T}$ at $c_{-}$.

Proposition 5.3. Under a first-order deformation of the hyperbolic structure on $M$,

$$
d V_{R}=\frac{1}{4}\left(\left\langle\operatorname{Re}\left(q_{-}\right), c_{-}^{\prime}\right\rangle+\left\langle\operatorname{Re}\left(q_{+}\right), c_{+}^{\prime}\right\rangle\right) .
$$

Proof of Theorem 1.2. Let $c:[0, d] \rightarrow \mathcal{T}$ be the geodesic segment parameterized at constant velocity 1 between $c_{-}$and $c_{+}$. Integrate the equation in the previous proposition with $c_{+}$replaced by $c(t), t \in[0, d]$, in Proposition 5.3. This shows that

$$
V_{R}\left(c_{-}, c_{+}\right)=\int_{t=0}^{d} \frac{1}{4}\left\langle q(t), c^{\prime}(t)\right\rangle d t,
$$

where $q(t)$ is the holomorphic quadratic differential equal to the Schwarzian differential of the identity between the Fuchsian complex projective structure obtained from Riemann uniformization from $c(t)$, and the quasifuchsian complex projective structure obtained by applying the Bers double uniformization theorem to $\left(c_{-}, c(t)\right)$.

The inequality in Corollary 5.2 shows that for all $t \in[0, d]$,

$$
\frac{1}{4}\left\langle q(t), c^{\prime}(t)\right\rangle \leq 3 \pi \sqrt{g-1},
$$

and the statement follows.

\section{The size of almost flat holomorphic disks}

In this section, we prove Theorem 1.5, giving an upper bound on the radius of holomorphic disk in $\mathcal{T}_{S}$ which are flat enough. The proof is based on a well-known upper bound on the curvature of $g_{W P}$ and on two key properties of the renormalized volume, as collected in the next lemma.

Lemma 6.1. Let $D$ be a holomorphic disk immersed in $\mathcal{T}_{S}$, with induced metric $q$, with center $c_{0}$. Consider the function $u: D \rightarrow \mathbb{R}$ defined by $u(c)=V_{R}\left(c_{0}, c\right)$ for all $c \in D$. Then

(1) $q$ has negative curvature,

(2) $\|d u\|_{q} \leq 3 \sqrt{\pi(g-1)}$,

(3) $\Delta_{q} u=-2$. 
Proof. The first point follows from the fact that the Weil-Petersson metric on $\mathcal{T}_{S}$ has negative sectional curvature [23] and from the Gauss formula, which indicates that the curvature of a holomorphic disk in a Kähler manifold is at most equal to the sectional curvature of the ambiant metric on its tangent space.

The second point follows from Proposition 3.10 and from Corollary 5.2.

For the third point recall that the renormalized volume $V_{R}\left(c_{-}, \cdot\right)$, considered as a function on $\mathcal{T}_{S}$, is a Kähler potential for the Weil-Petersson metric on $c T_{S}$; see e.g. $[21,22,14,16]$. In other terms:

$$
2 \partial \bar{\partial} V_{R}\left(c_{-}, \cdot\right)=i \omega_{W P} .
$$

But the restriction of $\partial \bar{\partial}$ to $D$ is $\partial \bar{\partial}$, so equal to $-(1 / 4) \Delta_{q}$, where $\Delta_{q}$ is the Laplace operator of $(D, q)$.

Lemma 6.2. There is continuous, increasing function $\phi:[0,1) \rightarrow \mathbb{R}_{\geq 0}$ with $\phi(0)=0$ and $\lim _{1} \phi=\infty$ as follows. Let $(D, q)$ be a Riemannian disk of center $c_{0}$, and let $u: D \rightarrow \mathbb{R}$ be a smooth function. Suppose that:

- the radius of $(D, q)$ is $\phi(\delta)$,

- $\Delta_{q} u=1$,

- the curvature $K_{q}$ of $q$ is in $[-1,0]$.

Then there is a point $x \in D$ where $\|d u\|_{q} \geq \delta$.

The precise value of the function $\phi$ can be obtained by solving a differential equation.

Proof. For $r \in(0, R]$ the geodesic disk $B(r)$ of center $c_{0}$ and radius $r$ is convex. We denote by $A(r)$ its area, by $L(r)$ the length of its boundary, by $\kappa(r)$ the total curvature of its boundary, and by $\bar{K}(r)$ the mean of its curvature. By definition, $\bar{K}(r) \in[-1,0]$ for all $r \in(0, R]$. Moreover:

- $A^{\prime}(r)=L(r)$,

- $L^{\prime}(r)=\kappa(r)$,

- $A(r) \bar{K}(r)=2 \pi-\kappa(r)$ by the Gauss-Bonnet theorem.

It follows that

$$
L^{\prime}(r)=2 \pi-\bar{K}(r) A(r) .
$$

Let $y(r)=A(r) / L(r)$. Then

$$
\begin{aligned}
y^{\prime}(r) & =\frac{A^{\prime}(r) L(r)-A(r) L^{\prime}(r)}{L(r)^{2}}=\frac{L(r)^{2}-A(r)(2 \pi-A(r) \bar{K}(r))}{L(r)^{2}} \\
& =1+\left(\bar{K}(r)-\frac{2 \pi}{A(r)}\right) y(r)^{2} .
\end{aligned}
$$

The initial condition is $\lim _{0} y=0$ since $A(r) \sim \pi r^{2}$ and $L(r) \sim 2 \pi r$ at 0 . Since $q$ has curvature in $[-1,0], A(r) \geq \pi r^{2}$ and $\bar{K}(r) \geq-1$ for all $r$, so that

$$
y^{\prime}(r) \geq 1-\left(1+\frac{2}{r^{2}}\right) y(r)^{2} .
$$

So $y(r) \geq y_{0}(r)$, where $y_{0}$ is the solution vanishing at 0 of the equation obtaining by taking the equality in (6.1). 
Let $\bar{u}(r)$ be the mean of $u$ over $\partial B(r)$. Then

$$
\bar{u}^{\prime}(r)=\frac{1}{L(r)} \int_{\partial B(r)} d u(n)=\frac{1}{L(r)} \int_{B(r)} \Delta_{q} u d a=\frac{A(r)}{L(r)}=y(r) .
$$

It follows that there exists a point at distance $r$ from $c$ where $\partial u / \partial r \geq y(r)$, and therefore where $\|d u\|_{q} \geq y(r) \geq y_{0}(r)$.

The lemma follows, with $\phi$ equal to the reciprocal of $y_{0}$.

Corollary 6.3. Let $\Delta_{0}, k, \delta>0$. Let $(D, q)$ be a Riemannian disk of center $c_{0}$, and let $u: D \rightarrow \mathbb{R}$ be a smooth function. Suppose that:

- the radius of $(D, q)$ is $\phi\left(k^{2} \delta / \Delta_{0}\right) / k$,

- $\Delta_{q} u=\Delta_{0}$,

- the curvature $K_{q}$ of $q$ is in $\left[-k^{2}, 0\right]$.

Then there is a point $x \in D$ where $\|d u\|_{q} \geq \delta$.

Proof. The statement is obtained by scaling the metric $q$ by a factor $k^{2}$ and the function $u$ by a factor $k^{2} / \Delta_{0}$ in Lemma 6.2.

Proof of Theorem 1.5. It follows directly from Lemma 6.1 and from Corollary 6.3. The function $\phi$ is inverse function of the solution of the differential equation

$$
y^{\prime}(r)=1-\left(1+\frac{2}{r^{2}}\right) y(r)^{2},
$$

which vanishes at 0 . An easy asymptotic analysis shows that $\lim _{\infty} y=1$, while $y^{\prime}(0)=$ $1 / 2$. It follows that $\phi$ is defined on $[0,1)$ with $\lim _{1} \phi=\infty$, and that $\phi^{\prime}(0)=2$.

\section{Acknowledgments}

The author would like to thank Juan Souto for useful conversations related to the results presented here, and Colin Guillarmou and Frédéric Paulin for providing relevant references necessary at some points of the arguments. Thanks also to Jiming Ma, Greg McShane, Sergiu Moroianu and to an anonymous referee for many helpful comments leading to improvement over the first version of this text. The work was partially supported by the A.N.R. through projects ETTT, ANR-09-BLAN-0116-01, and GeomEinstein, ANR-09-BLAN-0116-01.

\section{References}

[1] A. Besse, Einstein Manifolds, Springer, 1987.

[2] M. Bridgeman, Average bending of convex pleated planes in hyperbolic three-space, Invent. Math. 132(2) (1998), 381-391.

[3] J.F. Brock, The Weil-Petersson metric and volumes of 3-dimensional hyperbolic convex cores, J. Amer. Math. Soc. 16(3) (2003), 495-535 (electronic).

[4] Z. Djadli, C. Guillarmou, and M. Herzlich, Opérateurs géométriques, invariants conformes et variétés asymptotiquement hyperboliques, Panoramas et Synthèses [Panoramas and Syntheses], 26, Société Mathématique de France, Paris (2008), ISBN 978-2-85629-260-0.

[5] D. Dumas, Complex projective structures, in Handbook of Teichmüller theory. Vol. II, IRMA Lect. Math. Theor. Phys., 13 455-508, Eur. Math. Soc., Zürich, 2008.

[6] C.L. Epstein, Envelopes of horospheres and Weingarten surfaces in hyperbolic 3-space, 1984, Preprint.

[7] F.P. Gardiner and N. Lakic, Quasiconformal Teichmüller theory, Mathematical Surveys and Monographs, 76, American Mathematical Society, Providence, RI, 2000, ISBN 0-8218-1983-6. 
[8] C.R. Graham, Volume and area renormalizations for conformally compact Einstein metrics, in The Proceedings of the 19th Winter School "Geometry and Physics" (Srní, 1999), vol. 63, 31-42, 2000.

[9] C.R. Graham and J. M. Lee, Einstein metrics with prescribed conformal infinity on the ball, Adv. Math. 87 (1991) 186-225.

[10] C.R. Graham and E. Witten, Conformal anomaly of submanifold observables in AdS/CFT correspondence, Nucl. Phys. B 546(1-2) (1999), 52-64.

[11] C. Guillarmou and S. Moroianu, Chern-Simons line bundle on Teichmüller space, to appear, Geometry and Topology, arXiv:1102.1981 (2011).

[12] C. Guillarmou, S. Moroianu, and J.-M. Schlenker, The renormalized volume and uniformisation of conformal structures, arxiv:1211.6705 (2012).

[13] K. Krasnov, Holography and Riemann surfaces, Adv. Theor. Math. Phys. 4(4) (2000), 929-979.

[14] K. Krasnov and J.-M. Schlenker, On the renormalized volume of hyperbolic 3-manifolds, Commun. Math. Phys. 279(3) (2008), 637-668.

[15] —, A symplectic map between hyperbolic and complex Teichmüller theory (2008), arXiv:0806.0010. Duke Math. J. 150(2) (2009), 331-356.

[16] - The Weil-Petersson metric and the renormalized volume of hyperbolic 3-manifolds, in Handbook of Teichmüller theory. Volume III, IRMA Lect. Math. Theor. Phys., 17 779-819, Eur. Math. Soc., Zürich (2012).

[17] C.T. McMullen, The moduli space of Riemann surfaces is Kähler hyperbolic, Ann. Math. (2) 151(1) (2000), 327-357.

[18] S.J. Patterson and P. A. Perry, The divisor of Selberg's zeta function for Kleinian groups, Duke Math. J. 106(2) (2001), 321-390. Appendix A by Charles Epstein.

[19] I. Rivin and J.-M. Schlenker, The Schläfli formula in Einstein manifolds with boundary, Electron. Res. Announcements AMS 5 (1999), 18-23.

[20] — The Schläfli formula and Einstein manifolds (2000). arXiV:math.DG/0001176

[21] L. Takhtajan and P. Zograf, On uniformization of Riemann surfaces and the Weil-Petersson metric on the Teichmüller and Schottky spaces, Mat. Sb. 132 (1987) 303-320. English translation in Math. USSR Sb. 60 (1988), 297-313

[22] L.A. Takhtajan and L.-P. Teo, Liouville action and Weil-Petersson metric on deformation spaces, global Kleinian reciprocity and holography, Comm. Math. Phys. 239(1-2) (2003), $183-240$.

[23] S. Wolpert, Chern forms and the Riemann tensor for the moduli space of curves, Inventiones Math. 85(1) (1986), 119-145.

[24] S.A. Wolpert, Geodesic-length functions and the Weil-Petersson curvature tensor, J. Differential Geom. 91(2) (2012), 321-359.

[25] S.A. Wopert, The Weil-Petersson metric geometry, in Handbook of Teichmüller theory. Vol. II, IRMA Lect. Math. Theor. Phys., 13 47-64, Eur. Math. Soc., Zürich, 2008.

Mathematics Research Unit, University of Luxembourg, Campus Kirchberg, Blg, 6, rue

Richard Coudenhove-Kalergi, L-1359, Luxembourg, Luxembourg

E-mail address: jean-marc.schlenker@uni.lu 